\title{
Association Between Immunologic Markers And Cirrhosis In Individuals With Chronic Hepatitis B
}

llona Argirion ( $\square$ ilona.argirion@nih.gov )

National Cancer Institute

Ruth M. Pfeiffer

National Cancer Institute

Tram Kim Lam

National Cancer Institute

Thomas R. O'Brien

National Cancer Institute

Kelly Yu

National Cancer Institute

Katherine A. McGlynn

National Cancer Institute

Jessica L. Petrick

National Cancer Institute

Ligia Pinto

Frederick National Laboratory for Cancer Research, Biomedical Research, Inc

\section{Chien-Jen Chen}

Academia Sinica

Mei-Hsuan Lee

National Yang-Ming University

Allan Hildesheim

National Cancer Institute

Hwai-I Yang

Academia Sinica

Jill Koshiol

National Cancer Institute

Research Article

Keywords: cirrhosis, hepatitis B, circulating immunologic proteins, inflammation, prospective studies

Posted Date: June 11th, 2021

DOl: https://doi.org/10.21203/rs.3.rs-564386/v1

License: @ (1) This work is licensed under a Creative Commons Attribution 4.0 International License. Read Full License

Version of Record: A version of this preprint was published at Scientific Reports on November 15th, 2021. See the published version at https://doi.org/10.1038/s41598-021-00455-8. 


\section{Abstract}

Background: Host immune response and chronic inflammation associated with chronic hepatitis B virus (HBV) infection play a key role in the pathogenesis of liver diseases such as cirrhosis and hepatocellular carcinoma (HCC).

Methods: We sampled $175 \mathrm{HCC}, 117$ cirrhotic and 165 non-cirrhotic controls from a prospective cohort study of chronically HBV-infected individuals. Multivariable polytomous logistic regression and canonical discriminant analysis (CDA) were used to compare baseline plasma levels for 102 markers in individuals who developed cirrhosis vs. controls and those who developed HCC vs. cirrhosis. Leave-oneout cross validation was used to generate receiver operating characteristic curves to compare the predictive ability of marker groups.

Results: After multivariable adjustment, HGF (Q4v10R:3.74;p-trend=0.0001), SLAMF1 (Q4v10R:4.07;p-trend=0.0001), CSF1 (Q4v10R:3.00;p-trend=0.002), uPA (Q4v10R:3.36;p-trend=0.002), IL-8 (Q4v10R:2.83;p-trend=0.004), and OPG (Q4v1OR:2.44;ptrend $=0.005$ ) were all found to be associated with cirrhosis development compared to controls; these markers predicted cirrhosis with $69 \%$ accuracy. CDA analysis identified a nine marker model capable of predicting cirrhosis development with $79 \%$ accuracy. No markers were significantly different between HCC and cirrhotic participants.

Conclusion: This is the first prospective study to assess immunologic markers in relation to liver disease in chronically-HBV infected individuals. While validation in required, these findings highlight the importance of immunologic processes in HBV-related cirrhosis.

\section{Introduction}

The World Health Organization estimates that 257 million people worldwide are living with chronic hepatitis B virus (HBV) infection ${ }^{1}$. In spite of significant public health efforts over the past few decades, HBV-related liver cirrhosis and the increased risk of hepatocellular carcinoma (HCC) among infected persons remains a major health concern ${ }^{2}$. Liver disease etiology is heterogenous. Nevertheless, the estimated fractions of cirrhosis and HCC attributable to HBV infection are believed to be as high as $30 \%$ and $53 \%$, respectively, corresponding to over 200,000 annual deaths due to cirrhosis and 300,000 annual deaths due to $\mathrm{HCC}^{3}$.

Despite being at increased risk of liver disease, most individuals with HBV infection do not develop cirrhosis or HCC. The natural history of chronic HBV infection and subsequent disease, which remains to be fully elucidated, is impacted by both viral (HBV DNA levels, genotype and mutation patterns) and host-specific factors (age, sex, genetic polymorphisms, and immune status). Host immune response plays a critical role in the susceptibility to chronic HBV infection; $5-10 \%$ of adults with acute HBV infection fail to clear the virus and develop chronic disease due to inadequate adaptive immune response ${ }^{4}$. Studies on HBV pathogenesis in liver disease have largely been limited to experimental and small-scale clinical models, but consistently demonstrate the role of chronic inflammation in disease progression. A few studies have shown HBV specific T cells, chemokine-mediated neutrophil infiltrates, lymphocytes and natural killer cells to play a role in HBV-related liver damage ${ }^{5,6}$. This inflammatory microenvironment results in oxidative stress, ultimately promoting Kupffer cells to drive stellate activation through nuclear factor $\mathrm{KB}(\mathrm{NF}-\mathrm{KB})$, leading to progressive fibrosis and ultimately cirrhosis $^{6-9}$.

The role of circulating immunologic proteins have been investigated in prospective cohort studies in the context of HCC development, demonstrating the potential utility of markers such as C-reactive protein (CRP) ${ }^{10,11}$, interleukin-6 (IL-6) $)^{11,12}$, insulin-like growth factor binding protein-3 (IGFBP-3) $)^{13,14}$, and intercellular adhesion molecule 1 (ICAM-1) ${ }^{15}$ in predicting carcinogenesis. Nevertheless, no studies to our knowledge have comprehensively evaluated the role of circulating immunologic proteins on cirrhosis development in chronically HBV infected individuals. The morbidity and mortality associated with cirrhosis, and the accompanying risk of HCC among cirrhotic individuals are high. As such, assessing which circulating markers are associated with cirrhosis vs. chronic HBV infection and with HCC vs. cirrhosis may shed light and on the biological processes involved and potentially inform risk prediction. In this study, we utilize data from the Risk Evaluation of Viral Load Elevation and Associated Liver Disease/Cancer-Hepatitis B Virus (REVEAL-HBV) cohort to broadly evaluated the association between circulating immunologic markers and cirrhosis in chronically HBV infected participants.

\section{Methods}

\section{Study Population}

REVEAL-HBV is a longitudinal, community-based cohort study that was developed to better understand the natural history and risk factors of HCC among chronic HBV carriers ${ }^{16}$. In brief, between 1991-1992, 23,820 individuals aged 30-65 years were recruited across 
seven townships in Taiwan. After serological assessment of baseline samples, 4,155 participants were determined to be HBV surface antigen (HBsAg)-seropositive; among these individuals, 3,653 were seronegative for hepatitis $\mathrm{C}$ antibodies with no evidence of HCC. Covariates such as age, HBV genotype, smoking, alcohol use, body mass index (BMI), and history of diabetes were collected. REVEALHBV was approved by the Institutional Review Board of the College of Public Health of the National Taiwan University (Taipei, Taiwan), all participants provided informed consent, and the study was conducted in accordance with all relevant guidelines and regulation.

Development of cirrhosis and HCC were both closely tracked within the REVEAL-HBV cohort through biannual or annual exams until December 31, 2008. Cirrhosis was detected using high-resolution, real-time abdominal ultrasound as well as a quantitative scoring system reflective of liver surface features (normal, irregular, undulated), liver parenchymal texture (normal, heterogeneous, coarse), size of the intrahepatic blood vessel (normal, obscure, narrowing) and splenic size (normal, enlarged). After 1999, cirrhosis was further diagnosed through data linkage with the National Taiwan Health Insurance Database and confirmed by medical chart review. HCC diagnosis was similarly assessed through follow-up examination using ultrasound, a-fetoprotein testing, or data linkage with the Taiwan National Cancer Registry. Additional linkage to the national death certificate program was conducted and incident cases of HCC were verified through medical review of histopathological records; HCC case verification was obtained when a lesion was detected using at least two imaging techniques (abdominal ultrasonography, angiogram, or computed tomography) and/or one imaging technique and a serum a-fetoprotein level of $\geq 400 \mathrm{ng} / \mathrm{mL}$.

For this case-control analysis, 92 persons with cirrhosis and 129 chronic HBV carriers (i.e. controls) were matched to HCC cases on sex, age at sample collection, HBV DNA level ( $<10000$ or $\geq 10000$ copies $/ \mathrm{mL}$ ), and time from entry to sample collection $+/-2$ years; frequency matching on sex, age at sample collection and HBV DNA level ( $<10000$ or $\geq 10000$ copies $/ \mathrm{mL}$ ) was used to identify an additional 25 persons with cirrhosis and 36 controls. At the time of sample selection cirrhosis was assessed through 2004 and HCC through 2011; controls were not required to survive until the end of follow-up.

\section{Marker measurements}

Upon collection, all samples were stored at $70^{\circ} \mathrm{C}$ at the Academia Sinica in Taipei, Taiwan, until $250 \mu \mathrm{l}$ of heparin plasma were aliquoted onto 96 well plates and shipped to National Cancer Institute. Selected samples were from baseline or as near baseline as possible. 184 immunologic proteins were evaluated using the Olink (Uppsala, Sweden) inflammation and cardiometabolic panels, each panel requiring $1 \mu \mathrm{L}$ of sample. 48 blinded quality control samples from twelve patients with chronic HBV infection and twelve with chronic HCV infection were included on the panels. The limit of detection was achieved in $>90 \%$ of samples for all but 12 markers-which were excluded from analyses; an additional 69 markers were excluded due to intraclass correlation coefficients $<80 \%$ and one marker was removed due to a coefficient of variation $>25 \%$ (Supplementary Table 4). A total of 102 markers were included in the final analysis.

\section{Statistical Analysis}

Serological markers were evaluated as categorical variables and grouped in the following manner: for markers detectable in $\geq 75 \%$ of controls, quartiles were created based on values above the limit of detection (LLOD), with those at LLOD assigned to the lowest quartile. For markers detectable in $50-75 \%$ of controls, four categories were created with participants at LLOD being assigned to the lowest quartile, and the rest were divided into tertiles. For markers detectable in $25-50 \%$ of controls, tertiles were created with samples at LLOD comprising the lowest tertile and the remaining two categories calculated based on the median split value above LLOD. Finally, for markers detectable in $<25 \%$ of persons, binary coding was created based on those at vs. above LLOD.

Unconditional polytomous logistic regression models were used to assess the association between individual categorical circulating immunologic markers and stages of chronic HBV related liver disease, specifically: persons with cirrhosis compared to controls and HCC participants compared to persons with cirrhosis. The association between these markers and HCC vs. controls has been described previously ${ }^{17}$. Potential confounders were evaluated using a priori knowledge and backward selection. Final models included continuous age, sex, continuous years of follow-up, quartiles of HBV DNA (quartiles in controls: <300-12570, 12571-45919, 45920-229307, 229308 + copies $/ \mathrm{mL}$ ), serum alanine aminotransferase (ALT) $(<15,15-44, \geq 45 \mathrm{U} / \mathrm{L})$, alcohol, smoking, and HBV e-antigen (HBeAg). Statistical significance was determined after correcting for multiple testing using a Benjamini-Hochberg false discovery rate correction of $10 \%$. To address potential concerns regarding differences in duration of infection, we conducted a sensitivity analysis stratified by HBV DNA level using median cut-offs among controls. To assess the role of temporality on the association found between circulating markers and disease status, an additional sensitivity analysis stratified by median time to cirrhosis diagnosis among participants with cirrhosis who did not progress to HCC (6.57 years) was conducted. Statistical significance for these sensitivity analyses was determined using an $a=$ 0.05 . 
Canonical discriminant analysis (CDA) was used to discern linear groups of circulating immune markers found to be differentially expressed across the same comparison groups assessed by logistic regression models; statistical significance for backward selection

was determined using an $a=0.05$. Finally, leave-one-out cross validation ${ }^{18}$ was used to calculate the area under the receiver operating characteristic curves (AUCs) to evaluate and compare the predictive ability of three models: ${ }^{1}$ clinical/demographic characteristics alone (age, sex, years of follow-up, HBV viral load, ALT, drinking, smoking, and HBeAg), ${ }^{2}$ clinical/demographic variables plus the markers found to be significant in the logistic regression model, and ${ }^{3}$ clinical/demographic variables plus the markers found to be significant in the CDA. Separate figures were generated for the comparison between persons who developed cirrhosis vs. controls and persons who developed HCC vs. cirrhosis only. Statistical significance for the comparison between the curves was determined based on the DeLong, DeLong, and Clarke-Pearson method using an $a=0.05$. All statistical analyses were performed with SAS software version 9.4 (SAS Institute, Inc, Cary, NC).

\section{Results}

The median time from sample collection to cirrhosis development among the 117 persons with cirrhosis was 6.57 years (range: 0.3611.98); the median time from sample collection to HCC diagnosis was 10.50 years (range: $0.07-18.40$ ). There were no major differences noted in terms of sex distribution, alcohol consumption, smoking, family history of $\mathrm{HCC}$, or BMI across HCC cases, persons with cirrhosis and controls. HCC cases had higher baseline ALT levels and were more likely to be HBeAg positive compared to the other groups (Table 1). Approximately $62 \%$ (109/175) of participants who developed HCC had underlying cirrhosis. 
Table 1

Participants Characteristics

\begin{tabular}{|c|c|c|c|}
\hline \multicolumn{4}{|c|}{ REVEAL-HBV } \\
\hline Factor & HCC Cases & Cirrhosis Cases & Controls \\
\hline Total N & 175 & 117 & 165 \\
\hline$\%$ Male & $80.6 \%$ & $78.6 \%$ & $81.2 \%$ \\
\hline Median Age at Sample Date (Range) & $51(30-67)$ & $46(32-69)$ & $51(30-66)$ \\
\hline Median Year of Serum Collection (Range) & 1993 (1992-1997) & 1993 (1992-1997) & 1993 (1992-1998) \\
\hline Median Years of Follow-up (Range)* & $15.4(1.7-23.6)$ & $19.8(5.3-23.7)$ & $19.9(5.5-22.4)$ \\
\hline Median BMI (Range) & $24.4(17.0-37.7)$ & $23.8(16.4-32.6)$ & $23.4(17.2-33.4)$ \\
\hline \multicolumn{4}{|l|}{ ALT, N (\%) } \\
\hline$<15$ & $65(37.1)$ & $48(41.0)$ & $98(59.4)$ \\
\hline $15-44$ & $83(47.4)$ & $60(51.3)$ & $59(35.8)$ \\
\hline$\geq 45$ & $27(15.4)$ & $9(7.7)$ & $8(4.8)$ \\
\hline \multicolumn{4}{|l|}{ Smoking $^{\dagger}$} \\
\hline No & $103(58.9)$ & $71(60.7)$ & $108(65.5)$ \\
\hline Yes & $71(40.6)$ & $46(39.3)$ & $57(34.5)$ \\
\hline \multicolumn{4}{|l|}{ Alcohol drinking $^{\dagger}$} \\
\hline No & $140(80.0)$ & 104 (88.9) & $138(83.6)$ \\
\hline Yes & $34(19.4)$ & $13(11.1)$ & $26(15.8)$ \\
\hline \multicolumn{4}{|l|}{ Family history of HCC } \\
\hline No & 158 (90.3) & $112(95.7)$ & 151 (91.5) \\
\hline Yes & $17(9.7)$ & $5(4.3)$ & $14(8.5)$ \\
\hline \multicolumn{4}{|l|}{ HBeAg positive at baseline } \\
\hline No & 99 (56.6) & $91(77.8)$ & $146(88.5)$ \\
\hline Yes & $76(43.4)$ & $26(22.2)$ & 19 (11.5) \\
\hline $\begin{array}{l}\text { Median HBV DNA level (copies/ml) } \\
\text { at sample date }\end{array}$ & 1938060 & 141426 & 45920 \\
\hline
\end{tabular}

Assessment of individual markers using multivariable logistic regression yielded six proteins that were found to be positively associated with cirrhosis when compared to controls. Persons who developed cirrhosis were four times more likely to have elevated levels of signaling lymphocytic activation molecule 1 (SLAMF1) at baseline when compared to controls (odds ratio [OR] $]_{\mathrm{Q} 4 \mathrm{v} \text { Q1 }}=4.07$ [95\%Cl: $1.85-$ 8.99]); similar associations were found for hepatocyte growth factor (HGF) with an OR $\mathrm{Q}_{\mathrm{vv} . \mathrm{Q} 1}=3.74$ (95\%Cl:1.67-8.38), colony stimulating factor 1 (CSF1) with an $\mathrm{OR}_{\mathrm{Q} 4 \mathrm{v} . \mathrm{Q} 1}=3.00$ (95\% Cl: $\left.1.38-6.49\right)$, urokinase-type plasminogen activator (UPA) with an OR ${ }_{\mathrm{Q} 4 \mathrm{~V} . \mathrm{1}}=3.36$ (95\%Cl: 1.52-7.40), interleukin-8 (IL-8) with an OR $\mathrm{Q}_{\mathrm{Qv} . \mathrm{Q} 1}=2.83$ (95\% Cl: $\left.1.29-6.23\right)$, and OPG with an OR $\mathrm{Q}_{\mathrm{v} . \mathrm{Q} 1}=2.44$ (95\%Cl: $\left.1.14-5.22\right)(\mathrm{Table} 2)$. We did not find any markers to be significantly associated with HCC risk when compared to persons with cirrhosis after FDR correction (Supplementary Table 1). 
Table 2

Odds ratios* and $95 \%$ Cls for associations between selected markers and cirrhosis versus non-cirrhotic controls.

\begin{tabular}{|c|c|c|c|c|c|}
\hline \multirow[t]{3}{*}{ Analyte } & \multicolumn{5}{|l|}{ Cirrhosis vs control } \\
\hline & \multirow[b]{2}{*}{ Q2 v. Q1 } & \multicolumn{2}{|l|}{ OR $(95 \% \mathrm{Cl})$} & \multirow[t]{2}{*}{ P-trend } & \multirow[t]{2}{*}{ FDR-corrected p-trend } \\
\hline & & Q3 v. Q1 & Q4 v. Q1 & & \\
\hline HGF & $1.19(0.49-2.91)$ & $3.14(1.37-7.21)$ & $3.74(1.67-8.38)$ & 0.0001 & 0.007 \\
\hline SLAMF1 & $1.31(0.56-3.06)$ & $2.32(1.01-5.35)$ & $4.07(1.85-8.99)$ & 0.0001 & 0.007 \\
\hline CSF1 & $1.42(0.61-3.28)$ & $3.05(1.36-6.82)$ & $3.00(1.38-6.49)$ & 0.002 & 0.05 \\
\hline uPA & $1.69(0.72-3.96)$ & $2.14(0.94-4.85)$ & $3.36(1.52-7.40)$ & 0.002 & 0.05 \\
\hline IL8 & $1.70(0.76-3.84)$ & $2.95(1.36-6.38)$ & $2.83(1.29-6.23)$ & 0.004 & 0.08 \\
\hline OPG & $0.80(0.34-1.87)$ & $1.98(0.94-4.21)$ & $2.44(1.14-5.22)$ & 0.005 & 0.08 \\
\hline
\end{tabular}

The sensitivity analysis stratified by HBV DNA level at baseline yielded similar results as those reported in the primary analysis. All six markers were found to be significantly associated with cirrhosis among those with high ( $\geq 45,920$ copies/ml) viral levels. Among those with low HBV viral levels at baseline (<45,920 copies/ml) only the associations between HGF, SLAMF1, uPA, and cirrhosis were found to be statistically significant (Table 3 ). In assessing whether temporality plays a role in the observed associations, all six markers were significantly associated with cirrhosis development among those diagnosed $<6.57$ years after serum collection. Additionally, the effect sizes for these markers were larger within this subset when compared to the original analysis. HGF, SLAMF1, CSF1, and uPA were associated with cirrhosis among those diagnosed $\geq 6.57$ years after serum collection (Table 4 ).

Table 3

REVEAL-HBV odds ratios* and $95 \%$ Cls stratified by viral load

\begin{tabular}{|c|c|c|c|c|c|c|c|c|}
\hline \multirow[t]{3}{*}{ Analyte } & \multicolumn{4}{|c|}{ Low HBV Viral Level $(<45,920)$} & \multicolumn{4}{|c|}{ High HBV Viral Level $(\geq 45,920)$} \\
\hline & \multirow{2}{*}{$\begin{array}{l}\text { P- } \\
\text { trend }\end{array}$} & & \multicolumn{2}{|l|}{ OR $(95 \% \mathrm{Cl})$} & \multirow{2}{*}{$\begin{array}{l}\text { P- } \\
\text { trend }\end{array}$} & \multicolumn{3}{|c|}{ OR $(95 \% \mathrm{Cl})$} \\
\hline & & Q2 v. Q1 & Q3 v. Q1 & Q4 v. Q1 & & Q2 v. Q1 & Q3 v. Q1 & Q4 v. Q1 \\
\hline \multicolumn{9}{|c|}{ Cirrhosis v. Control } \\
\hline HGF & 0.03 & $\begin{array}{l}1.85(0.45- \\
7.65)\end{array}$ & $\begin{array}{l}3.25(0.79- \\
13.35)\end{array}$ & $\begin{array}{l}4.26(1.09- \\
16.58)\end{array}$ & 0.004 & $\begin{array}{l}0.99(0.30- \\
3.27)\end{array}$ & $\begin{array}{l}2.97(1.02- \\
8.69)\end{array}$ & $\begin{array}{l}3.40(1.22- \\
9.52)\end{array}$ \\
\hline SLAMF1 & 0.02 & $\begin{array}{l}4.91(1.07- \\
22.48)\end{array}$ & $\begin{array}{l}3.56(0.77- \\
16.5)\end{array}$ & $\begin{array}{l}6.80(1.56- \\
29.59)\end{array}$ & 0.003 & $\begin{array}{l}0.61(0.20- \\
1.84)\end{array}$ & $\begin{array}{l}2.26(0.76- \\
6.74)\end{array}$ & $\begin{array}{l}3.03(1.12- \\
8.21)\end{array}$ \\
\hline CSF1 & 0.36 & $\begin{array}{l}1.66(0.45- \\
6.14)\end{array}$ & $\begin{array}{l}3.21(0.85- \\
12.12)\end{array}$ & $\begin{array}{l}1.64(0.43- \\
6.23)\end{array}$ & 0.002 & $\begin{array}{l}1.23(0.40- \\
3.78)\end{array}$ & $\begin{array}{l}2.62(0.93- \\
7.40)\end{array}$ & $\begin{array}{l}3.97(1.48- \\
10.67)\end{array}$ \\
\hline UPA & 0.04 & $\begin{array}{l}2.74(0.58- \\
12.94)\end{array}$ & $\begin{array}{l}2.77(0.59- \\
12.94)\end{array}$ & $\begin{array}{l}4.87(1.10- \\
21.65)\end{array}$ & 0.04 & $\begin{array}{l}1.75(0.59- \\
5.21)\end{array}$ & $\begin{array}{l}1.92(0.70- \\
5.28)\end{array}$ & $\begin{array}{l}2.97(1.11- \\
7.99)\end{array}$ \\
\hline IL8 & 0.41 & $\begin{array}{l}1.45(0.38- \\
5.62)\end{array}$ & $\begin{array}{l}1.89(0.54- \\
6.61)\end{array}$ & $\begin{array}{l}1.74(0.45- \\
6.72)\end{array}$ & 0.01 & $\begin{array}{l}1.54(0.54- \\
4.40)\end{array}$ & $\begin{array}{l}3.64(1.30- \\
10.20)\end{array}$ & $\begin{array}{l}3.03(1.11- \\
8.22)\end{array}$ \\
\hline OPG & 0.09 & $\begin{array}{l}1.29(0.34- \\
4.86)\end{array}$ & $\begin{array}{l}2.05(0.6- \\
7.02)\end{array}$ & $\begin{array}{l}2.71(0.75- \\
9.8)\end{array}$ & 0.03 & $\begin{array}{l}0.53(0.17- \\
1.68)\end{array}$ & $\begin{array}{l}2.08(0.77- \\
5.63)\end{array}$ & $\begin{array}{l}2.24(0.83- \\
6.02)\end{array}$ \\
\hline
\end{tabular}


Table 4

REVEAL-HBV odds ratios* and $95 \%$ Cls stratified by time to cirrhosis diagnosis

\begin{tabular}{|c|c|c|c|c|c|c|c|c|}
\hline \multirow[t]{3}{*}{ Analyte } & \multicolumn{4}{|c|}{ Early cirrhosis diagnosis (< 6.57 years) } & \multicolumn{4}{|c|}{ Late cirrhosis diagnosis ( $\geq 6.57$ years) } \\
\hline & \multirow[t]{2}{*}{$\begin{array}{l}\text { P- } \\
\text { trend }\end{array}$} & \multicolumn{3}{|c|}{ OR $(95 \% \mathrm{Cl})$} & \multirow[t]{2}{*}{$\begin{array}{l}\mathrm{P}- \\
\text { trend }\end{array}$} & \multicolumn{3}{|c|}{$\begin{array}{l}\text { OR }(95 \% \\
\mathrm{Cl})\end{array}$} \\
\hline & & Q2 v. Q1 & Q3 v. Q1 & Q4 v. Q1 & & Q2 v. Q1 & Q3 v. Q1 & Q4 v. Q1 \\
\hline \multicolumn{9}{|c|}{ Cirrhosis v. Control } \\
\hline HGF & 0.0006 & $0.88(0.23,3.36)$ & $2.95(0.94,9.3)$ & $4.48(1.51,13.27)$ & 0.007 & $\begin{array}{l}1.56 \\
(0.54,4.51)\end{array}$ & $\begin{array}{l}3.56 \\
(1.31,9.69)\end{array}$ & $\begin{array}{l}3.32 \\
(1.21,9.09)\end{array}$ \\
\hline SLAMF1 & 0.001 & $2.67(0.74,9.67)$ & $3.32(0.92,11.97)$ & $6.83(2.01,23.22)$ & 0.003 & $\begin{array}{l}0.78 \\
(0.28,2.23)\end{array}$ & $\begin{array}{l}1.96 \\
(0.74,5.22)\end{array}$ & $\begin{array}{l}3.14 \\
(1.26,7.81)\end{array}$ \\
\hline CSF1 & 0.0008 & $\begin{array}{l}2.50 \\
(0.69,9.05)\end{array}$ & $\begin{array}{l}6.37 \\
(1.91,21.27)\end{array}$ & $\begin{array}{l}6.20 \\
(1.90,20.26)\end{array}$ & 0.04 & $\begin{array}{l}1.09 \\
(0.41,2.87)\end{array}$ & $\begin{array}{l}1.90 \\
(0.74,4.90)\end{array}$ & $\begin{array}{l}2.21 \\
(0.91,5.39)\end{array}$ \\
\hline uPA & 0.010 & $\begin{array}{l}2.18 \\
(0.70,6.80)\end{array}$ & $1.04(0.30,3.56)$ & $\begin{array}{l}4.10 \\
(1.45,11.55)\end{array}$ & 0.02 & $\begin{array}{l}1.44 \\
(0.50,4.15)\end{array}$ & $\begin{array}{l}3.06 \\
(1.16,8.07)\end{array}$ & $\begin{array}{l}2.60 \\
(0.97,6.97)\end{array}$ \\
\hline IL8 & 0.006 & $\begin{array}{l}1.24 \\
(0.41,3.72)\end{array}$ & $2.79(1.04,7.47)$ & $3.37(1.22,9.32)$ & 0.05 & $\begin{array}{l}2.15 \\
(0.78,5.90)\end{array}$ & $\begin{array}{l}3.33 \\
(1.25,8.86)\end{array}$ & $\begin{array}{l}2.48 \\
(0.91,6.74)\end{array}$ \\
\hline OPG & 0.0008 & $\begin{array}{l}0.41 \\
(0.09,1.84)\end{array}$ & $2.69(0.93,7.74)$ & $\begin{array}{l}4.09 \\
(1.44,11.64)\end{array}$ & 0.20 & $\begin{array}{l}0.95 \\
(0.37,2.44)\end{array}$ & $\begin{array}{l}1.64 \\
(0.68,4.00)\end{array}$ & $\begin{array}{l}1.60 \\
(0.63,4.02)\end{array}$ \\
\hline
\end{tabular}

CDA isolated nine markers that together differentiated between those who developed cirrhosis vs. controls; these markers included HGF and SLAMF1, as well as angiogenin (ANG), insulin-like growth factor-binding protein 3 (IGFBP3), plasma serine protease inhibitor (SERPINA5), eotaxin (CCL11), two C-X-C motif chemokines (CXCL11 and CXCL9), and STAM-binding protein (STAMBP). ROC curves for this comparison grouping yielded an area under the curve (AUC) of $63.3 \%$ for the clinical/demographic variables only model. The addition of the six markers identified in the logistic regression model improved predictability to $68.5 \%$, while the nine marker model from the CDA analysis yielded an AUC of 79.3\% (Fig. 1); ROC contrast test results found AUC curves for the marker inclusive models to significantly improved predictability when compared to the clinical/demographic only model, with a p-value $\leq 0.0001$.

CDA analysis for the comparison between those who developed HCC vs. those who developed cirrhosis but not HCC yielded 11 markers that together were found to be significantly different between these groups, including: IGFBP3, SLAMF1, eukaryotic translation initiation factor 4E-binding protein 1 (4E-BP1), caspase-8 (CASP-8), T cell surface glycoprotein CD6 isoform (CD6), three interleukin (IL-8, IL-13, IL24), monocyte chemotactic protein 3 (MCP-3), stem cell factor (SCF), and TNF-related apoptosis-inducing ligand (TRAIL). Due to the lack of significant markers found in the logistic regression analysis for this comparison, only two ROC curves were generated; no significant difference ( $p$-value $=0.07$ ) was found between the clinical/demographic variable only model $(A U C=86.6 \%)$ and the model that included the 11 markers from the CDA analysis (AUC $=88.5 \%)($ Fig. 1$)$.

\section{Discussion}

This is the first study to our knowledge to prospectively evaluate a broad list of immunologic markers as they relate to the development of cirrhosis in HBV infected participants. Herein, we identified six individual proteins (HGF, SLAMF, CSF1, UPA, IL-8, OPG) to be significantly positively associated with the development of cirrhosis; these markers were found to be particularly strongly associated among individuals who developed cirrhosis within seven years after sample collection. Lack of an association between these immunologic markers and HCC suggests that these markers may influence progression to cirrhosis among HBV infected individuals, but not further progression to HCC. Compared to clinical/demographic variables alone, the addition of the six markers improved predictability of cirrhosis development by about $6 \%$ (63\% vs. $69 \%$ ). In addition to evaluating the markers individually, we assessed how well a combination of markers might discriminate between individuals with chronic HBV infection who go on to develop cirrhosis versus those who do not. This analysis identified a nine-protein set comprised of ANG, IGFBP3, SERPINA5, CCL11, CXCL11, CXCL9, HGF, SLAMF1, and STAMBP that in addition to clinical/demographic variables was able to predict cirrhosis development with $79 \%$ accuracy in a cross-validation model. 
Our study found increased levels of HGF, CSF1, and uPA-three markers believed to be associated with liver regeneration-to be associated with greater odds of developing clinical cirrhosis. HGF, functions to regulate cellular motility and development and has previously been shown to be elevated in cirrhotic patients ${ }^{19,20}$. In the context of repeated injury due to an infectious agent such as HBV, continued regeneration of liver cells can result in the overgrowth of fibroblasts that ultimately result in the development of cirrhosis ${ }^{21}$. Albeit through a different method, CSF1 controls macrophage numbers that facilitate the hepatic innate immune defense and ultimately support hepatocyte proliferation following injury. Elevated circulating CSF1 levels have been associated with rapid liver regrowth in transplant and donor patients ${ }^{22,23}$ as well as acute liver injury, cirrhosis, and HCC $^{23}$. Finally, uPA is a serine protease that has been implicated in the direct and indirect degradation of matrix proteins and is believed to have a bidirectional effect in the liver. Increased expression of the uPA cell-surface binding protein results in increased hepatic uPA in the early stages of liver regeneration, nonetheless in later phases, uPA derived plasmin leads to the suppression of hepatocyte growth ${ }^{24-26}$. While uPA has previously been reported to be elevated in fibrotic/cirrhotic patients ${ }^{27}$, its dual effects on hepatocytes has recently spurred interest in its utility in gene therapy ${ }^{28,29}$.

In accordance with HBV mediated inflammation, we found elevated levels of IL-8 and SLAMF1 to be associated with the development of clinical cirrhosis. IL-8 is a CXC chemokine that has been well established as a major factor in acute inflammation. Serum IL-8 levels have been associated with disease progression in chronically infected hepatitis C virus (HCV) and HBV patients ${ }^{30,31}$. Increased levels of IL-8 in both the liver and in circulation have been reported in alcoholic hepatitis, ischemia-reperfusion injury, and fibrosis/cirrhosis ${ }^{32-35}$. Similarly, CXCL11 and CXCL9 were only marginally significant after FDR correction in logistic regression analyses but were both identified as differentiating markers of cirrhosis in CDA analyses. Elevated levels of these proteins, as seen in our analysis, have previously been reported in liver diseases (both with and without infectious etiology) and functionally linked to inflammation, hepatic injury and fibrosis ${ }^{36-38}$. SLAMF1 (recently renamed CD150) modulates TGF- $\beta$ signaling and has been proposed to function in regulating the epithelial-mesenchymal transition associated with tissue repair, fibrogenesis and carcinogenesis ${ }^{39-41}$. Elevated levels of SLAMF1 have been associated with TGF- $\beta 1$ upregulation, inflammation and more recently cirrhosis ${ }^{42}$.

Cirrhosis can be associated with a number of metabolic disorders including osteopenia and osteoporosis, insulin resistance, and malnutrition ${ }^{43-45}$. Receptor activator of nuclear factor KB ligand (RANKL) and OPG work together to maintain bone homeostasis by binding to the receptor RANK. Together, the OPG/RANKL system plays a role in linking the immune system and bone metabolism. Previous studies have demonstrated high levels of OPG and low levels of RANKL in cirrhotic patients compared to those with noncirrhotic chronic liver disease and control populations ${ }^{46}$; this phenomenon is believed to be due to a compensatory response to halt bone loss within affected persons ${ }^{47}$. In accordance with this literature, but for the first time in chronically infected HBV participants, we found elevated levels of OPG to be associated with increased odds of cirrhosis development. In contrast, lower levels of IGFBP3 were associated with cirrhosis development. In CDA analysis, IGFBP-3 was determined to differentiate persons with cirrhosis from controls; decreased levels of IGFBP-3 in persons with cirrhosis have previously been described and attributed to the fact that IGFBP-3 is predominantly synthesized by hepatocytes and reportedly associated with severity of liver dysfunction ${ }^{48,49}$.

A major strength of the study was that REVEAL-HBV is a well characterized prospective cohort study that provides a unique opportunity to evaluate the association between immunologic markers and development of cirrhosis in chronically HBV infected individuals; nevertheless, this study does have several limitations. Because cirrhosis was not diagnosed using data linkage with the National Taiwan Health Insurance Database until after 1999, there is a potential for under-diagnosis of cirrhosis from 1991-1999. However, cohort participants received an abdominal ultrasonography every 6-12 months, substantially reducing the risk of under-diagnosis even without linkage to the insurance database. Additionally, we did not have data on the use of anti-HBV therapies, which could potentially mask associations; however, antiviral therapy was not reimbursed by the Taiwanese universal health care system through 2003 , which encompasses most of the study period, and after 2003 was only provided to high-risk patients under stringent criteria. Finally, due to the limited sample size, analyses between serum markers and disease may have been underpowered in stratified analyses.

Better understanding the natural history of disease among high risk patients with chronic HBV may offer important insights into prevention and treatment of liver disease. In this novel, comprehensive evaluation of circulating immunologic proteins, we identified several markers that were found to significantly distinguish and predict cirrhosis development, particularly among those diagnosed within seven years of blood draw. While screening protocols for patients chronically infected with HBV do exist, the addition of serum biomarkers may aid in risk stratification to further enhance these procedures. Although this study requires replication, these findings highlight the importance of immunologic and proliferative pathways in HBV-related liver disease.

\section{Declarations}


Financial support: This study was supported by the Academia Sinica and Ministry of Science and Technology (101-2314-B-001-005MY3) in Taiwan. This work was also supported by general funds from the Intramural Research Program of the National Institutes of Health, National Cancer Institute, Division of Cancer Epidemiology and Genetics.

Conflict of Interest: No authors have any relevant conflicts of interest.

Author Contributions

I.A determined the methodology, conducted the formal analysis, and wrote the manuscript. R.M.P aided in overseeing the statistical methodology as well as reviewing and editing the manuscript. T.K.L and L.P helped in reviewing and editing the manuscript. T.R.O, K.A.M, J.L.P, and A.H aided in conceptualizing the study, as well as reviewing and editing the manuscript. K.Y helped with data curation, as well as reviewing and editing the manuscript. C.C, M.L, and H.Y aided in data curation, investigation, funding acquisition and project administration. J.K led the conceptualization of the study, acquired funding, supervised the analysis, and aided in reviewing and editing the manuscript.

\section{References}

1 Organization, W. H. Global hepatitis report 2017. (World Health Organization, 2017).

2 hepatology, E. A. F. T. S. O. T. L. J. J. o. EASL 2017 Clinical Practice Guidelines on the management of hepatitis B virus infection. 67, 370-398 (2017).

3 Perz, J. F., Armstrong, G. L., Farrington, L. A., Hutin, Y. J. \& Bell, B. P. J. J. o. h. The contributions of hepatitis B virus and hepatitis C virus infections to cirrhosis and primary liver cancer worldwide. 45, 529-538 (2006).

4 Chen, C. J., Yang, H. I. J. J. o. g. \& hepatology. Natural history of chronic hepatitis B REVEALed. 26, 628-638 (2011).

5 Edmunds, W., Medley, G., Nokes, D., Hall, A. \& Whittle, H. J. P. o. t. R. S. o. L. S. B. B. S. The influence of age on the development of the hepatitis B carrier state. 253, 197-201 (1993).

6 Suhail, M. et al. Potential mechanisms of hepatitis B virus induced liver injury. 20, 12462 (2014).

7 Ganem, D. \& Prince, A. M. J. N. E. J. o. M. Hepatitis B virus infection-natural history and clinical consequences. 350, 1118-1129 (2004).

8 Fattovich, G., Stroffolini, T., Zagni, I. \& Donato, F. J. G. Hepatocellular carcinoma in cirrhosis: incidence and risk factors. 127, S35-S50 (2004).

9 Luedde, T., Schwabe, R. F. J. N. r. G. \& hepatology. NF-KB in the liver-linking injury, fibrosis and hepatocellular carcinoma. 8, 108-118 (2011).

10 Chen, W. et al. Association between C-reactive protein, incident liver cancer, and chronic liver disease mortality in the Linxian Nutrition Intervention Trials: a nested case-control study. 24, 386-392 (2015).

11 Aleksandrova, K. et al. Inflammatory and metabolic biomarkers and risk of liver and biliary tract cancer. 60, 858-871 (2014).

12 Ohishi, W. et al. Serum interleukin-6 associated with hepatocellular carcinoma risk: A nested case-control study. 134, 154-163 (2014).

13 Adachi, Y. et al. Insulin-like growth factor-related components and the risk of liver cancer in a nested case-control study. 37, 1512515132 (2016).

14 Major, J. et al. Insulin-like growth factors and liver cancer risk in male smokers. 103, 1089-1092 (2010).

15 Chen, V. L. et al. Soluble intercellular adhesion molecule-1 is associated with hepatocellular carcinoma risk: multiplex analysis of serum markers. 7, 1-9 (2017).

16 Chen, C. J. \& Yang, H. I. Natural history of chronic hepatitis B REVEALed. J Gastroenterol Hepato/ 26, 628-638, doi:10.1111/j.1440-

1746.2011.06695.x (2011).

17 Koshiol, J. et al. (Submitted). 
18 Molinaro, A. M., Simon, R. \& Pfeiffer, R. M. Prediction error estimation: a comparison of resampling methods. Bioinformatics 21, 33013307, doi:10.1093/bioinformatics/bti499 (2005).

19 Costantini, S. et al. Cancer biomarker profiling in patients with chronic hepatitis C virus, liver cirrhosis and hepatocellular carcinoma. 29, 2163-2168 (2013).

20 Costantini, S. et al. Serum cytokine levels as putative prognostic markers in the progression of chronic HCV hepatitis to cirrhosis. European cytokine network 21, 251-256, doi:10.1684/ecn.2010.0214 (2010).

$21 \mathrm{Xie}, \mathrm{Q}$. et al. Overexpression of HGF promotes HBV-induced hepatocellular carcinoma progression and is an effective indicator for Met-targeting therapy. 4, 247-260 (2013).

22 Matsumoto, K. et al. Serial changes of serum growth factor levels and liver regeneration after partial hepatectomy in healthy humans. 14, 20877-20889 (2013).

23 Stutchfield, B. M. et al. CSF1 restores innate immunity after liver injury in mice and serum levels indicate outcomes of patients with acute liver failure. 149, 1896-1909. e1814 (2015).

24 Andreasen, P. A., Kjøller, L., Christensen, L. \& Duffy, M. J. J. I. j. O. C. The urokinase-type plasminogen activator system in cancer metastasis: a review. 72, 1-22 (1997).

25 De Petro, G. et al. Expression of urokinase-type plasminogen activator (u-PA), u-PA receptor, and tissue-type PA messenger RNAs in human hepatocellular carcinoma. 58, 2234-2239 (1998).

26 Chan, C.-F. et al. Evaluation of nuclear factor-kB, urokinase-type plasminogen activator, and HBx and their clinicopathological significance in hepatocellular carcinoma. 10, 4140-4149 (2004).

27 Bidgoli, S. A. \& Zaverhei, M. D. J. I. J. o. C. R. Differential Expression of uPA in Chronic Hepatitis B and C, Liver Cirrhosis and Hepatocellular Carcinoma: Comparison. 3, 25-32 (2007).

28 Salgado, S. et al. Liver cirrhosis is reverted by urokinase-type plasminogen activator gene therapy. 2, 545-551 (2000).

29 Iredale, J. P. J. G. A cut above the rest? MMP-8 and liver fibrosis gene therapy. 126, 1199-1201 (2004).

30 Polyak, S. J., Khabar, K. S., Rezeiq, M. \& Gretch, D. R. J. J. o. v. Elevated levels of interleukin-8 in serum are associated with hepatitis C virus infection and resistance to interferon therapy. 75, 6209-6211 (2001).

31 Yang, K. et al. Enhanced levels of interleukin-8 are associated with hepatitis B virus infection and resistance to interferon-alpha therapy. 15, 21286-21298 (2014).

32 Jaeschke, H. J. A. J. o. P.-G. \& Physiology, L. Mechanisms of Liver Injury. II. Mechanisms of neutrophil-induced liver cell injury during hepatic ischemia-reperfusion and other acute inflammatory conditions. 290, G1083-G1088 (2006).

33 Jaeschke, H. J. A. Neutrophil-mediated tissue injury in alcoholic hepatitis. 27, 23-27 (2002).

34 Dominguez, M. et al. Hepatic expression of CXC chemokines predicts portal hypertension and survival in patients with alcoholic hepatitis. 136, 1639-1650 (2009).

35 Zimmermann, H. W. et al. Interleukin-8 is activated in patients with chronic liver diseases and associated with hepatic macrophage accumulation in human liver fibrosis. 6, e21381 (2011).

36 Larrubia, J. R., Benito-Martinez, S., Calvino, M., Sanz-de-Villalobos, E. \& Parra-Cid, T. Role of chemokines and their receptors in viral persistence and liver damage during chronic hepatitis C virus infection. World J Gastroentero/ 14, 7149-7159, doi:10.3748/wjg.14.7149 (2008).

37 Zeremski, M. et al. Intrahepatic levels of CXCR3-associated chemokines correlate with liver inflammation and fibrosis in chronic hepatitis C. Hepatology 48, 1440-1450, doi:10.1002/hep.22500 (2008). 
38 Apolinario, A. et al. Increased expression of T cell chemokines and their receptors in chronic hepatitis C: relationship with the histological activity of liver disease. Am J Gastroentero/ 97, 2861-2870, doi:10.1111/j.1572-0241.2002.07054.x (2002).

39 Barbara, N. P., Wrana, J. L. \& Letarte, M. J. J. o. B. C. Endoglin is an accessory protein that interacts with the signaling receptor complex of multiple members of the transforming growth factor- $\beta$ superfamily. 274, 584-594 (1999).

$40 \mathrm{Li}, \mathrm{C}$. et al. CD105 antagonizes the inhibitory signaling of transforming growth factor $\beta$ I on human vascular endothelial cells. 14, 5564 (2000).

$41 \mathrm{Li}$ C. et al. TNFa down-regulates CD105 expression in vascular endothelial cells: a comparative study with TGFß1. (2003).

42 Yagmur, E. et al. Elevation of endoglin (CD105) concentrations in serum of patients with liver cirrhosis and carcinoma. 19, 755-761 (2007).

43 Leslie, W. D., Bernstein, C. N. \& Leboff, M. S. J. G. AGA technical review on osteoporosis in hepatic disorders. 125, 941-966 (2003).

44 Huisman, E. J., Trip, E. J., Siersema, P. D., van Hoek, B. \& van Erpecum, K. J. Protein energy malnutrition predicts complications in liver cirrhosis. Eur J Gastroenterol Hepatol 23, 982-989, doi:10.1097/MEG.0b013e32834aa4bb (2011).

45 Goral, V., Atalay, R. \& Kucukoner, M. Insulin resistance in liver cirrhosis. Hepatogastroenterology 57, $309-315$ (2010).

46 Moschen, A. R. et al. The RANKL/OPG system and bone mineral density in patients with chronic liver disease. 43, 973-983 (2005).

47 López-Larramona, G., Lucendo, A. J., González-Castillo, S. \& Tenias, J. M. J. W. j. o. h. Hepatic osteodystrophy: an important matter for consideration in chronic liver disease. 3, 300 (2011).

48 Nedic, O., Nikolic, J. A., Prisic, S., Acimovic, J. \& hajdukovic-Dragojlovic, L. Reactivity of IGF binding protein-3 isoforms towards concanavalin A in healthy adults and subjects with cirrhosis. Addict Bio/ 8, 81-88, doi:10.1080/1355621031000069927 (2003).

49 Wu, Y. L., Ye, J., Zhang, S., Zhong, J. \& Xi, R. P. Clinical significance of serum IGF-I, IGF-II and IGFBP-3 in liver cirrhosis. World J Gastroenterol 10, 2740-2743, doi:10.3748/wjg.v10.i18.2740 (2004).

\section{Figures}




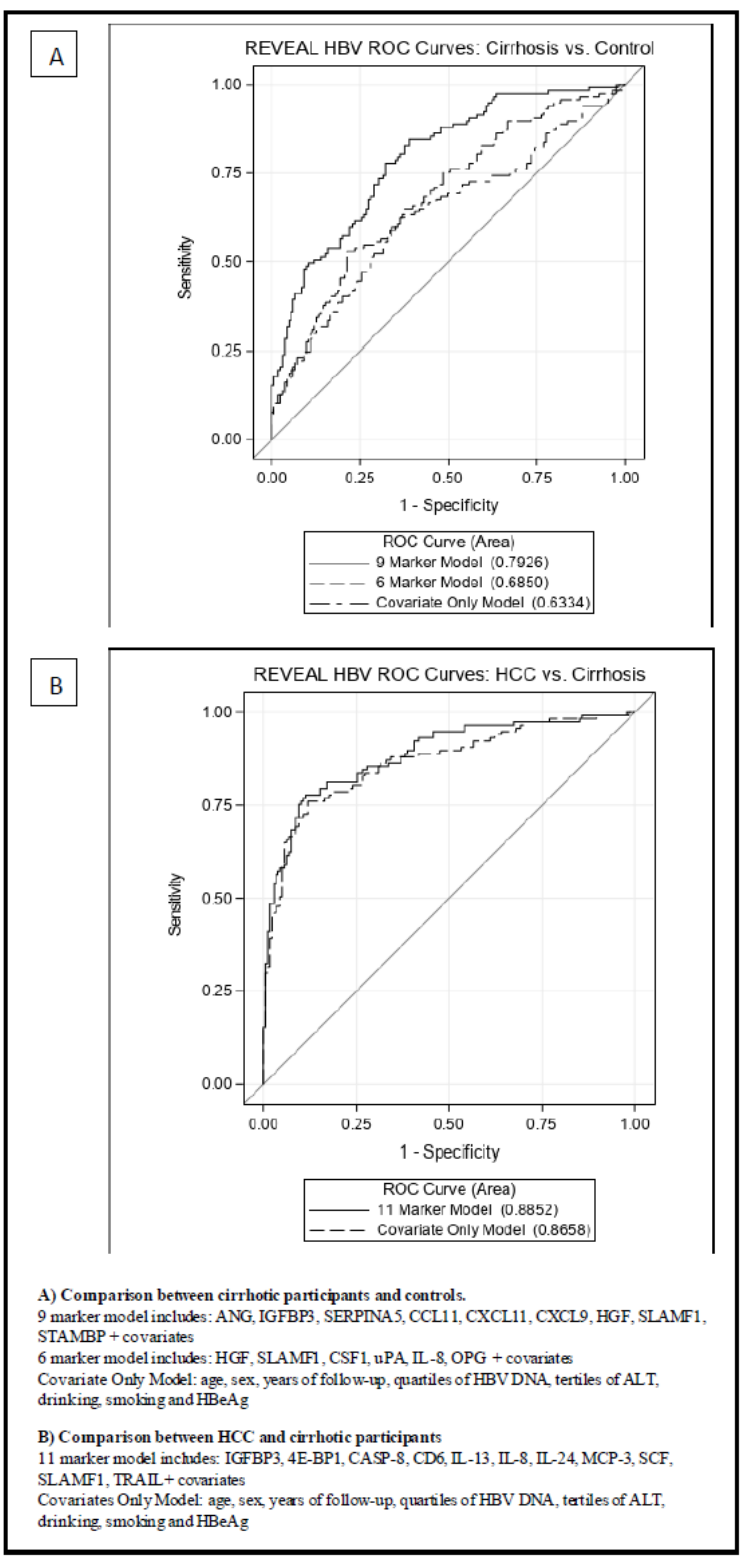

\section{Figure 1}

Receiver operating characteristic (ROC) curves comparing predictive models

\section{Supplementary Files}

This is a list of supplementary files associated with this preprint. Click to download.

- SupplementaryTables.docx 